\title{
Comparison of analytical functions used to describe topside electron density profiles with satellite data
}

\author{
Carlo Fonda, Pierdavide Coïsson, Bruno Nava and Sandro M. Radicella \\ The Abdus Salam International Centre for Theoretical Physics (ICTP), Trieste, Italy
}

\begin{abstract}
Electron density models of the ionosphere use different analytical formulations for the electron density vertical profile in the topside. The present paper compares some single-layer topside analytical descriptions (Chapman, Epstein, modified Epstein used in the NeQuick model) with experimental topside profiles obtained from measurements of IK19 and ISIS2 satellites. The limits of height range and shape for each formulation are described and analyzed and suggestions for the use of multiple layers solution to reproduce experimental results are given.
\end{abstract}

Key words topside ionosphere - ionospheric modeling - ionospheric layer formulation - topside soundings

\section{Introduction}

Different kinds of analytical functions are being used to model the electron density profile of the various ionospheric layers. Since the experimental information of the topside electron density profile is very scarce, this part of the modeled profiles has been less tested against measured data.

In the last few years a relatively large amount of topside electron density profiles has been made available to the scientific community through the Internet allowing to test models (Coïsson et al., 2002) and the different mathematical representations used.

This work analyzes the ability of some ionospheric layers analytical formulation to reproduce

Mailing address: Carlo Fonda, The Abdus Salam International Centre for Theoretical Physics (ICTP), Strada Costiera 11,34014 Trieste, Italy; e-mail: cfonda@ictp.it the experimental profiles measured by some old satellites which carried topside sounders: the Russian Intercosmos-19 (IK19) and the Canadian-American International Satellite for Ionosphere Studies 2 (ISIS2).

Some analytical aspects (derivative, inflection point and scale parameter) of common layer functions are investigated here in order to find better ways to model the lower part of the ionosphere topside.

\section{Layer functions for the topside}

Some simple layer functions have been chosen to perform the comparisons: Epstein, Chapman and other derived formulations. They are characterized by a scale parameter which determines the thickness of the layer.

Epstein layer function

$$
\operatorname{Epstein}\left(h, B_{2}\right)=\frac{4 \exp \left(h / B_{2}\right)}{\left(1+\exp \left(h / B_{2}\right)\right)^{2}}
$$

$h$ being the height relative to the $F 2$ maximum and $B_{2}$ the scale parameter for the layer. 

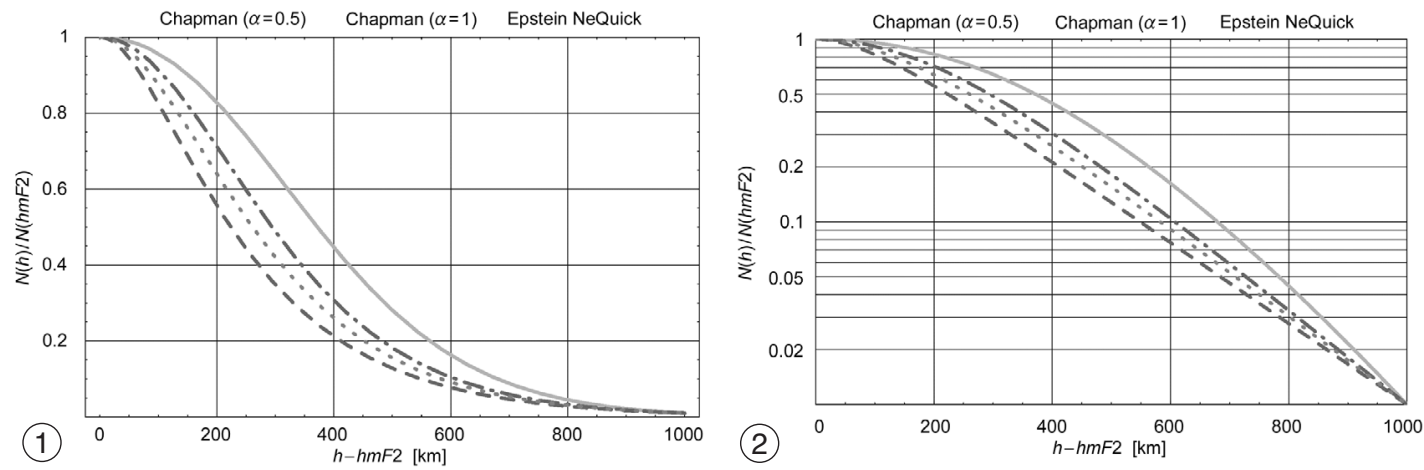

Fig. 1. Profile shapes for the different functions with intersection at $1000 \mathrm{~km}$ above $h m F 2$ : Chapman-alpha dashed, Chapman-beta dotted, Epstein dash-dotted, NeQuick solid gray. Linear scale.

Fig. 2. Profile shapes for the different functions with intersection at $1000 \mathrm{~km}$ above $h m F 2$ : Chapman-alpha dashed, Chapman-beta dotted, Epstein dash-dotted, NeQuick solid gray. Logarithmic scale.

«Modified Epstein» layer function

$$
\begin{gathered}
\operatorname{NeQuick}\left(h, H_{0}\right)=\frac{4 \exp k}{(1+\exp k)^{2}} \\
k=\frac{h}{H_{0}}\left(1+\frac{12.5 h}{0.125 h+100 H_{0}}\right)
\end{gathered}
$$

$h$ being the height relative to the $F 2$ maximum and $H_{0}$ the scale parameter for the layer. It is used in the NeQuick model (Radicella and Leitinger, 2001).

\section{Chapman family of layer functions}

$\operatorname{Chapman}(h, k, \alpha)=\exp \left[\alpha\left(1-\exp \left(-\frac{h}{k}\right)-\frac{h}{k}\right)\right]$

$h$ being the height relative to the $F 2$ maximum, $k$ the scale parameter for the layer and $\alpha$ the shape parameter. This function is usually called according to the value of $\alpha$ : Chapman-alpha if $\alpha=0.5$ and Chapman-beta if $\alpha=1$.

Examples of the described functions shapes are shown in fig. 1 in linear scale and in fig. 2 in logarithmic scale. All the profiles have been made to have an electron density of $0.001 \mathrm{NmF} 2$ at $1000 \mathrm{~km}$ above $h m F 2$.

\section{Data from IK19 satellite}

The first set of data is the one from the IK19 satellite. This satellite was on an elliptical orbit with $74^{\circ}$ inclination and it had perigee at $500 \mathrm{~km}$ and apogee at $980 \mathrm{~km}$ (Pulinets, 1989). The IK19 topside profiles cover height ranges form about $200 \mathrm{~km}$ up to about $750 \mathrm{~km}$ over the $F 2$ peak. It is a data set which allows to analyze the lower part of the topside. The data available are from the high solar activity period March 1979-December 1980 .

$\alpha$-Chapman, $\beta$-Chapman, Epstein and modified Epstein (NeQuick) layer functions were used to fit 7138 experimental electron density profiles derived from IK19 on board topside sounder. The fit was performed using least square methods, some examples are presented in figs. 3, 4 and 5. In addition the following parameters have also been evaluated: 1) height of the inflection point; 2) absolute difference between the integral of the fitting function and the integral of the experimental profile, numerically obtained.

\subsection{Analysis of integrals}

The four considered modeling functions have been analyzed, comparing for each profile the absolute difference $\varepsilon$ between the integral of 

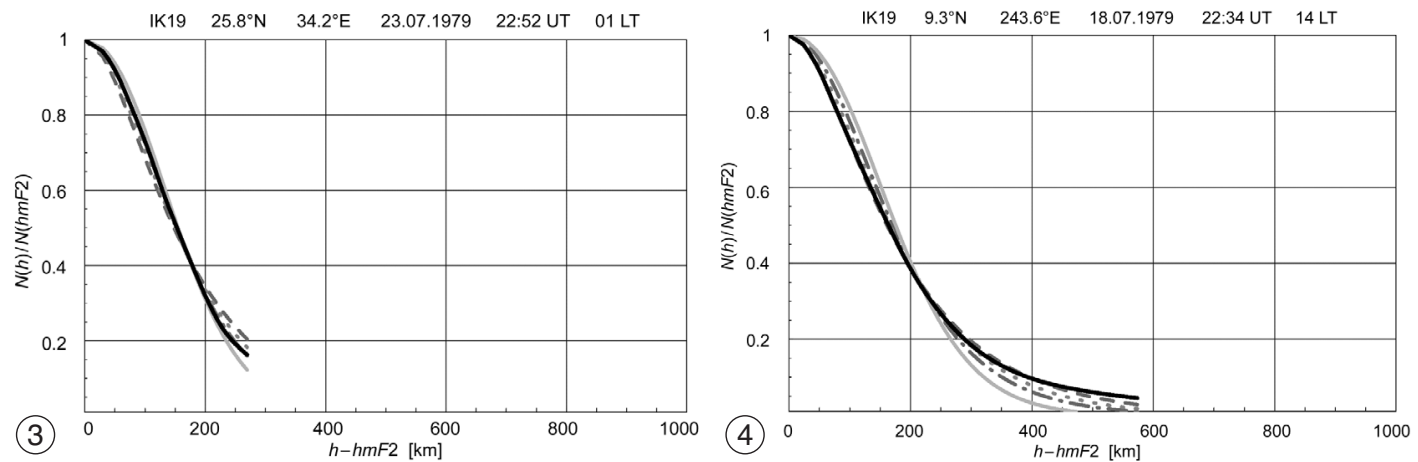

Fig. 3. IK19 experimental profile (solid black) and the fitted functions: Chapman-alpha dashed, Chapman-beta dotted, Epstein dash-dotted, NeQuick solid gray.

Fig. 4. IK19 experimental profile (solid black) and the fitted functions: Chapman-alpha dashed, Chapman-beta dotted, Epstein dash-dotted, NeQuick solid gray.
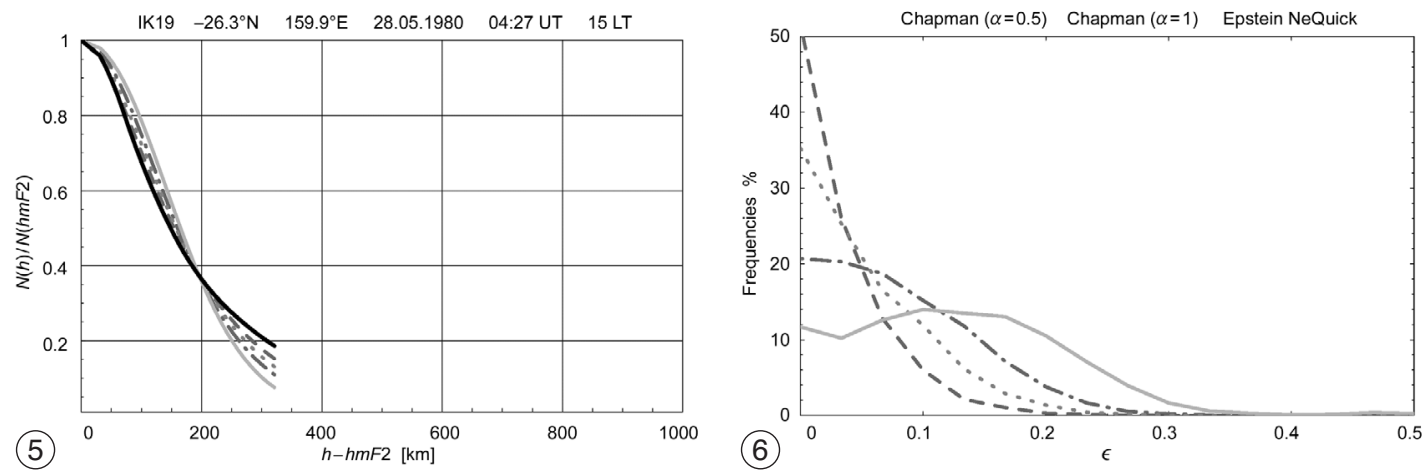

Fig. 5. IK19 experimental profile (solid black) and the fitted functions: Chapman-alpha dashed, Chapman-beta dotted, Epstein dash-dotted, NeQuick solid gray.

Fig. 6. Distribution of $\varepsilon$ for all IK19 profiles. Chapman-alpha dashed, Chapman-beta dotted, Epstein dash-dotted, NeQuick solid gray.

the fitting function and the integral of the experimental profile. This criterion has been used to evaluate how well the shape of these layer functions reproduce the behavior of experimental profiles, in order to identify which function is better able to model this part of the topside.

The obtained distribution, presented in fig. 6 , shows that a Chapman function with the shape parameter $\alpha=0.5$ gives th e best result, as confirmed by simply looking at most of the profile plots.

\subsection{Analysis of inflection points}

The height of the inflection point is used as an additional criterion to evaluate the capability of a function to reproduce the shape of the lowest part 

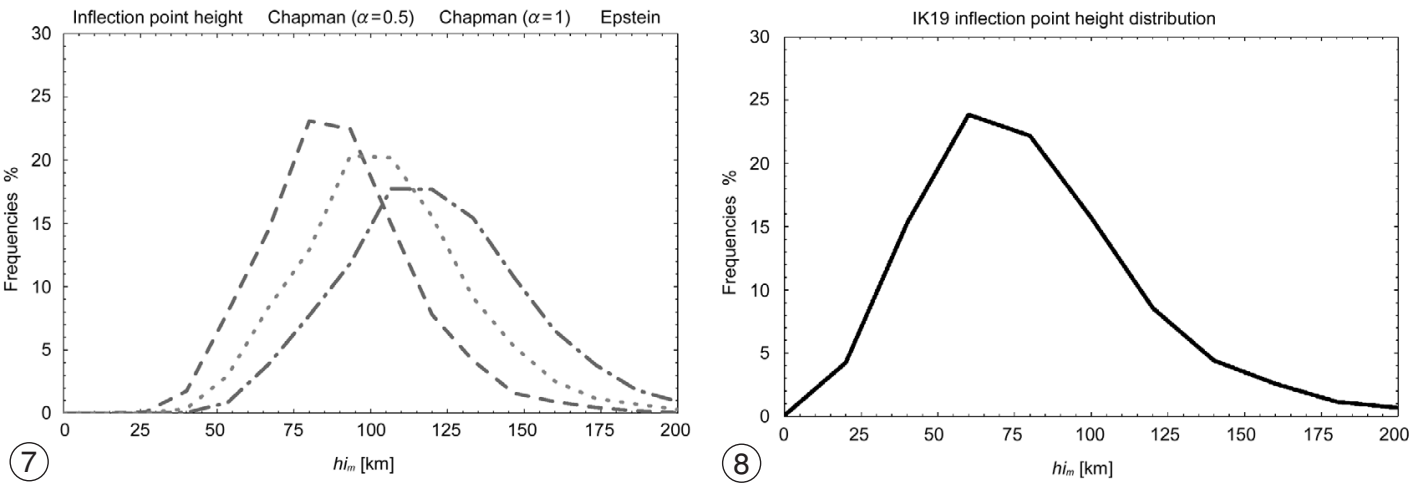

Fig. 7. Distribution of inflection point heights of fitted IK19 profiles: Chapman-alpha dashed, Chapman-beta dotted, Epstein dash-dotted.

Fig. 8. Distribution of inflection point heights of IK19 profiles.
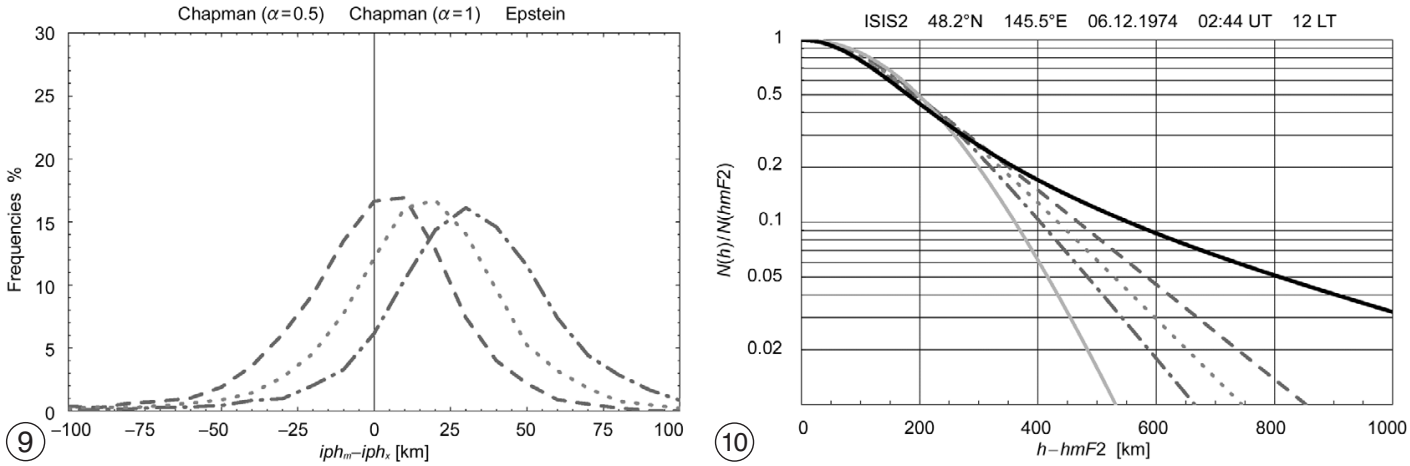

Fig. 9. Distribution of difference between experimental and fitted inflection point heights of IK19 profiles. Chapman-alpha dashed, Chapman-beta dotted, Epstein dash-dotted.

Fig. 10. ISIS2 experimental profile (solid black) and the fitted functions: Chapman-alpha dashed, Chapmanbeta dotted, Epstein dash-dotted, NeQuick solid gray.

of the topside (IK19 profiles usually have a maximum height in the order of $500 \mathrm{~km}$ above the $F 2$ peak). The distribution of these heights (fig. 7), compared to the positions of the inflection points as computed from the experimental profiles (fig. 8), again shows a better performance of the Chapman-alpha with respect to the other functions (fig. 9). The inflection points cannot be analytically determined for the NeQuick topside function and they are not included in such analysis.

\section{Data from ISIS2 satellite}

The ISIS2 satellite was in operation for more than a decade, it had a circular orbit at $1400 \mathrm{~km}$ with inclination $88^{\circ}$ (Bilitza et al., 2002, 2003; Huang et al., 2002). The same method of profile fitting has been applied to the experimental data set derived from ISIS2 satellite, some examples are shown in figs. 10, 11 and 12 . The results are consistent with those ob- 

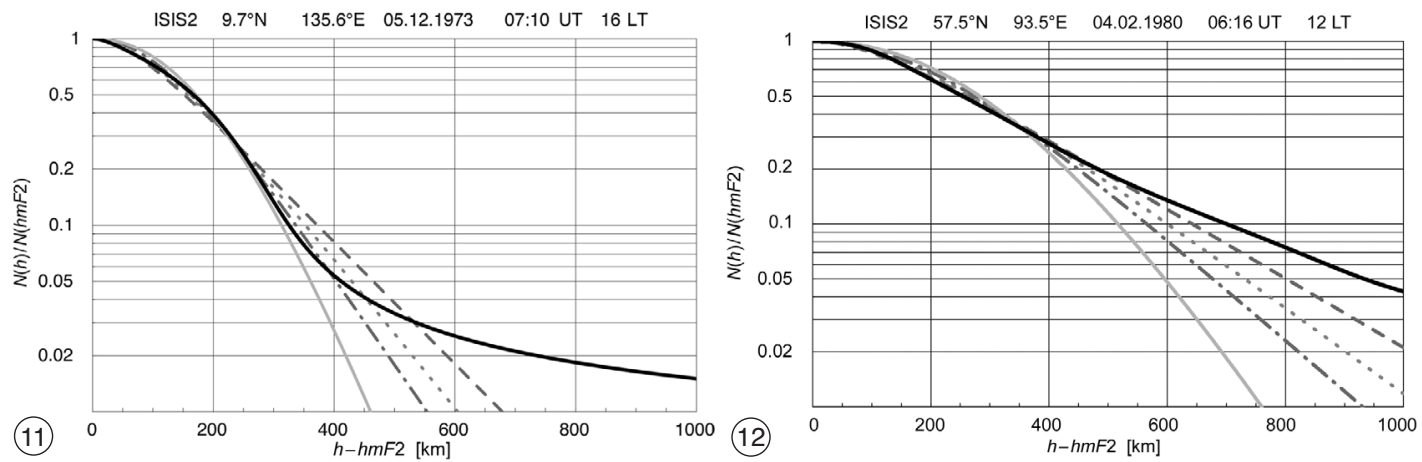

Fig. 11. ISIS2 experimental profile (solid black) and the fitted functions: Chapman-alpha dashed, Chapmanbeta dotted, Epstein dash-dotted, NeQuick solid gray.

Fig. 12. ISIS 2 experimental profile (solid black) and the fitted functions: Chapman-alpha dashed, Chapmanbeta dotted, Epstein dash-dotted, NeQuick solid gray.

tained from IK19 profiles. The main difference being the average maximum height of the profiles, higher for ISIS2, these results show better the behavior of model functions in a higher part of the topside (range of height: 400-1000 km above the $F 2$ peak).

The fits analysis also shows that the layer function used for the lower topside cannot be used «as it is» for this higher range of heights, but a new and different 2-layers topside formulation, at least, is needed in order to better reproduce the experimental shape.

The three selected profiles shown here are plotted with a logarithmic vertical scale to clearly indicate the different behavior of the model functions and the experimental profiles for this higher range of heights.

\section{Conclusions}

A few analytical aspects of two commonused topside layer functions, with some variants were studied with a comparison among them and experimental profiles, using a least square fit approach. It appeared that all the considered functions have difficulties in reproducing experimental data in the required range of height. It is also evident that a different analyt- ical formulation is needed in order to reproduce the relevant part of the topside. This new formulation may possibly involve a Chapman layer function with $\alpha<0.5$ for heights lower than $400 \mathrm{~km}$ above the $F 2$ maximum and should use a different functional approach for the higher part, up to $1000 \mathrm{~km}$ above $h m F 2$.

\section{REFERENCES}

Bilitza, D., X. Huang, B. Reinish and R.F. Benson (2002): TOPIST - Automated processing of ISIS topside ionograms, in Proceedings of 2002 Ionospheric Effects Symposium, 333-340.

Bilitza, D., B. Reinish, R. Benson, J. Grebowsky, N. PAPISTASHVILI, X. HuANG, W. Schar and K. Hills (2003): Online database of satellite sounder and insitu measurements covering two solar cycles, Adv. Space Res., 31 (3), 769-774.

CoÏsson, P., S.M. Radicella and B. NAVA (2002): Comparisons of experimental topside electron concentration profiles with IRI and NeQuick models, Ann. Geophysics, 45 (1), 111-116.

Huang, X., B.W. Reinisch, D. Bilitza and R.F. Benson (2002): Electron density profiles of the topside ionosphere, Ann. Geophysics, 45 (1), 125-130.

Pulinets, S.A. (1989): Prospect of topside sounding, in WITS Handbook (SCOSTEP Publishing, Urbana, IL), vol. 2, ch. 3, 99-127.

RADICELLA, S.M. and R. LEITINGER (2001): The evolution of the DGR approach to model electron density profiles, Adv. Space Res., 27 (1), 35-40. 